\title{
Conocimientos, actitudes y prácticas del consumo de sustancias psicoactivas en estudiantes de enfermería de una Universidad privada de Barranquilla 2010-2011
}

\section{Knowledge, attitudes and practices psychoactive substance nursing student a private University in 2010-2011 Barranquilla}

\section{Conhecimentos, atitudes e práticas do consumo de substâncias psicoativas em estudantes de enfermagem de uma universidade privada de Barranquilla 2010-2011}

\author{
Herminia Rosa Castellón-Montenegro', Cecilia Rocha-Rodríguez², Lina Montes-Rodríguez ${ }^{3}$, \\ Gloria Elena Lastre-Amell ${ }^{4}$
}

Forma de citar: H. R. Castellón, C. Rocha, 1. Montes, G. E. Lastre, "Conocimientos, actitudes y prácticas del consumo de sustancias psicoactivas en estudiantes de enfermería de una Universidad privada de Barranquilla 20102011”, Respuestas, vol. 20, no. 1, pp. 67-83, 2015.

Recibido:

14 de Mayo 2014

Aceptado:

24 de Octubre 2014
${ }^{1}$ Magister en Salud Pública herminiacastellon@unimetro.edu.co

Universidad Metropolitana Barranquilla- Colombia

${ }^{2}$ Especialista en Salud Familiar y Docencia Universitaria

Universidad Metropolitana Barranquilla- Colombia

${ }^{3}$ Especialista en Desarrollo Familiar Universidad Metropolitana Barranquilla- Colombia

${ }^{4}$ Magister en Salud Pública Universidad Metropolitana Barranquilla- Colombia

\section{Resumen:}

Objetivos: Determinar los conocimientos actitudes y prácticas relacionados con el consumo de sustancias psicoactivas en estudiantes de enfermería de una universidad privada del distrito de Barranquilla. Metodología: información obtenida directamente de la aplicación de la encuesta de 37 preguntas la cual fue diseñada por un médico toxicólogo, docente del área de farmacología de la División de Salud de la Universidad del Norte a los estudiantes de los distintos semestres para un total de 198. Este proceso se llevó a cabo en tres momentos: la socialización del estudio a los estudiantes y docentes de cada acompañantes; aplicación encuesta y por último, análisis y procesamiento de los resultados. Resultados: de los 198 estudiantes, menos del 70\% de ellos considera que las sustancias mencionadas son drogas, a pesar que el total de ellas son consideradas como tal. Más del $80 \%$ de las estudiantes conoce que las drogas producen efectos perjudiciales para la salud, a excepción de la benzodiacepina, orlasteina y LSD cuyos conocimientos son muy escasos. En general (mas del 88\%), de los estudiantes rechazan todo tipo de intencion de legalizar la produccion, trafico o consumo de Sustancias Psicoactivas (SPA), y apoyan todo esfuerzo del gobierno en erradicarlas y establecer programas que frenen su consumo. Del total de los estudiantes, aproximadamente un 97\% de ellos no han hecho uso de sustancias psicoactivas, a excepción del alcohol y de la nicotina. Conclusiones: Este estudio revela el importante papel que desempeñan los programas y escuelas de enfermería frente a la problemática de consumo de SPA, en la medida, en que los semestres son más complejos se observa un notable cambio de actitud y un nivel de conocimientos superior. La tarea de la educación en SPA es vital para concertar acciones interinstitucionales, interdisciplinares e intersectoriales para hacer promoción, prevención, tratamiento, rehabilitación y reinserción social de sujetos vulnerables y consumidores de sustancias psicoactivas en nuestra región.

Palabras claves: Actitudes, Conocimientos, Drogas, Educación, Enfermería, Practicas, Prevención, Sustancias Psicoactivas. 
No. 1

Enero - Junio 2015 ISSN 0122-820X

\begin{abstract}
Objectives: To determine the knowledge, attitudes and practices related to the use of psychoactive drugs in nursing students from a private university district of Barranquilla. Method: Information obtained directly from the application of the survey of 37 questions which was designed by a medical toxicologist, professor of pharmacology area of the Health Division of the Universidad del Norte to students of different semesters for a total of 198. This process took place in three stages: the socialization of study students and teachers of each escort, survey implementation and finally, analysis and processing of the results. Results: Of the 198 students, less than $70 \%$ of them feel that the above substances are drugs, although the total of them considered as such. Over $80 \%$ of the students know that drugs produce adverse health effects, except for benzodiazepine, and LSD Orlasteina whose skills are scarce. In general (over 88\%), our students reject all kinds of intention to legalize the production, trafficking or consumption of Psychoactive Substances (PSS), and support all efforts to eradicate government and establish programs to curb consumption. Of all students, about $97 \%$ of them have not made use of psychoactive substances, with the exception of alcohol and nicotine. Conclusion: This study reveals the important role of nursing programs and schools face the problem of consumption of PSS, as the semesters are more complex there is a remarkable change of attitude and a higher level of knowledge. The task of education is vital to arrange PSS agency actions, interdisciplinary and intersectorial for promotion, prevention, treatment, rehabilitation and social reintegration of vulnerable subjects and consumers of psychoactive substances in our region.
\end{abstract}

Keywords: Attitudes, Knowledge, Drugs, Education, Nursing, Practices, Prevention, Psychoactive Substances.

\title{
Resumo:
}

Objetivos: Determinar os conhecimentos atitudes e práticas relacionados com o consumo de substâncias psicoativas em estudantes de enfermagem de uma universidade privada do distrito de Barranquilla. Metodologia: Informação obtida diretamente da aplicação da pesquisa de opinião de 37 perguntas, a qual foi desenhada por um médico toxicólogo, docente da área de farmacologia da Divisão de Saúde da Universidad del Norte aos estudantes dos distintos semestres para um total de 198. Este processo foi realizado em três momentos: a socialização do estudo aos estudantes e docentes de cada acompanhante; aplicação pesquisa de opinião e por último, análise e processamento dos resultados. Resultados: dos 198 estudantes, menos do $70 \%$ deles considera que as sustâncias mencionadas são drogas, a pesar que o total delas são consideradas como tal. Mais do $80 \%$ das estudantes conhece que as drogas produzem efeitos prejudiciais para a saúde, a exceção da benzodiacepina, orlasteina e LSD cujos conhecimentos são muito escassos. Em geral (mais do 88\%), dos estudantes rejeitam todo tipo de intenção de legalizar a produção, trafego ou consumo de Substâncias Psicoativas (SPA), e apoiam todo esforço do governo em erradicar e estabelecer programas que frenem seu consumo. Do total dos estudantes, aproximadamente um $97 \%$ deles não tem feito uso de substâncias psicoativas, a exceção do álcool e da nicotina. Conclusões: Este estudo revela o importante papel que desempenham os programas e escolas de enfermagem frente à problemática de consumo de SPA, na medida, em que os semestres são mais complexos se observa uma notável mudança de atitude e um nível de conhecimentos superior. A tarefa da educação em SPA é vital para concertar ações interinstitucionais, interdisciplinares e inter-setoriais para fazer promoção, prevenção, tratamento, reabilitação e reinserção social de sujeitos vulneráveis e consumidores de substâncias psicoativas em nossa região.

Palavras chaves: Atitudes, Conhecimentos, Drogas, Educação, Enfermagem, Práticas, Prevenção, Substâncias Psicoativas. 


\section{Introducción}

En nuestro país el uso de sustancias psicoactivas plantea nuevos retos en el presente siglo. A la presencia de sustancias psicoactivas legales ya tradicionales como el alcohol, tabaco, e ilegales como la marihuana, cocaína, bazuco y drogas emergentes como la ketamina y el popper, causando gran alarma social y en los ámbitos de la salud indicando que estamos enfrentándonos al inicio de una epidemia del consumo de estas sustancias con todas las implicaciones de salud pública y sociales que el uso de estas drogas trae [1].

El consumo de sustancias psicoactivas, se considera un fenómeno creciente en el contexto mundial, y se convierte en un problema relevante de salud pública; Según el estudio Nacional de Consumo de Sustancias Psicoactivas en Colombia, 2008, al igual que en la gran mayoría de los países del mundo la marihuana es la sustancia ilícita de mayor consumo en Colombia, el 8\% dicen haber consumido esta sustancia, y el mayor consumo está en personas entre 18 y 24 años con un 5\%, el segundo lugar lo ocupa la cocaína, en la que un $2.5 \%$ de los encuestados dicen haberla consumido [2]. En consecuencia el Programa de Enfermería de la Universidad privada de Barranquilla, confirma la decisión de acoger las Políticas de la CICAD/OEA, para la reducción de las Sustancias Psicoactivas, a través de planes y proyectos, cuyos actores principales son los docentes y estudiantes formados con competencias para trabajar en la reducción de la demanda de la drogas a nivel intersectorial e interdisciplinaria. La reducción de la demanda de drogas es una labor con un gran potencial para la profesión de enfermería, la cual debe tener liderazgo y aplicar los conocimientos científicos y técnicos disponibles, promocionando estilos de vida saludables, reflejados en la atención a la persona, familia y comunidad en el contexto sociocultural.
El profesional de enfermería deberá desarrollar estrategias para prevenir el uso y abuso de drogas, actuando sobre los factores de riesgo y promoviendo la unión familiar. Actuando en primera instancia en el fomento de la salud, fortaleciendo los factores protectores, a fin de mejorar la autoestima.

De acuerdo a lo anterior esta investigación tuvo como objetivo determinar los conocimientos actitudes y prácticas relacionados con el consumo de sustancias psicoactivas en estudiantes de enfermería de una universidad privada del distrito de Barranquilla. De la misma forma determinar los aspectos socio demográficos de los estudiantes de enfermería sujetos del estudio, los conocimientos relacionados con el consumo, actitudes y practicas relacionados con el consumo de sustancias psicoactivas.

\section{Materiales y métodos}

Se enmarcó en un estudio descriptivo transversal, con muestreo aleatorio simple, la muestra estuvo conformada por 198 estudiantes. La fuente de información primaria se obtuvo de la aplicación de la encuesta, esta constó de 37 preguntas la cual fue diseñada por un médico toxicólogo, docente del área de farmacología de la División de Salud de la Universidad del Norte [3]. Las preguntas de la encuesta están distribuidas en aspectos sociodemográficos:(edad, sexo, preferencia sexual, número de hermanos, hijo número, estrato social, estado civil, cohabitantes, ocupación) variable de conocimiento actitudes y prácticas sobre el consumo se sustancias psicoactivas. Las consideraciones éticas que se tuvieron en cuenta fueron la Resolución 008430 de 1993 del Ministerio de Salud De Colombia, sobre los lineamientos éticos para estudios con seres humanos y el Código de ética de Enfermería [4], [5], además se socializó y se entregó el consentimiento informado a los estudiantes para ser firmado, una vez que se iniciara la aplicación de la
Enero - Junio 2015 ISSN 0122-820X PP: $67-83$ 
encuesta garantizando la confidencialidad y el anonimato de los datos, también se solicitó permiso al autor del instrumento para su aprobación de aplicación al igual que al comité de ética para la realización del estudio.

Después se procedió a aplicar la encuesta a cada estudiante que deseara participar voluntariamente al estudio que cumpliera con los criterios de inclusión (estudiantes de enfermería de primer a octavo semestre, que hayan firmado el consentimiento informado y los estudiantes menores de 18 años que deseen voluntariamente participar requirieron del asentimiento y la autorización de los padres), y exclusión (estudiantes que no deseen voluntariamente participar) este proceso tuvo una duración de 10 a 15 minuto, seguidamente se verifica cada encuesta para identificar su totalidad de diligenciamiento en todas las preguntas de la encuesta. El procesamiento de la información fue tabulada en una matriz de Excel, y en un software de SPSS versión 18; el análisis estadístico se realizó en frecuencia y porcentajes y se presentará mediante tablas y graficas de barras.

\section{Resultados y análisis}

A continuación se presenta los análisis de los resultados de las variables del estudio sociodemográfico:

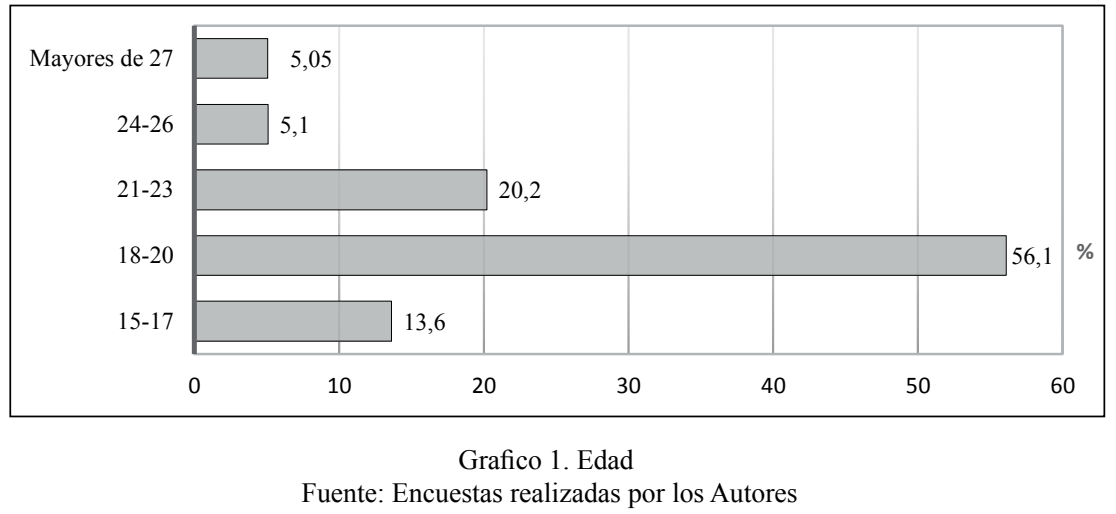

Los datos Sociodemográficos obtenidos mediante encuesta aplicada se identificó que el 56.1\% de los estudiantes encuestados se sitúa dentro del rango de edades comprendido entre los 18 y 20 años, cabe de anotar que el 13.6\% de los estudiantes en edades entre 15-17 años es decir menores de edad.

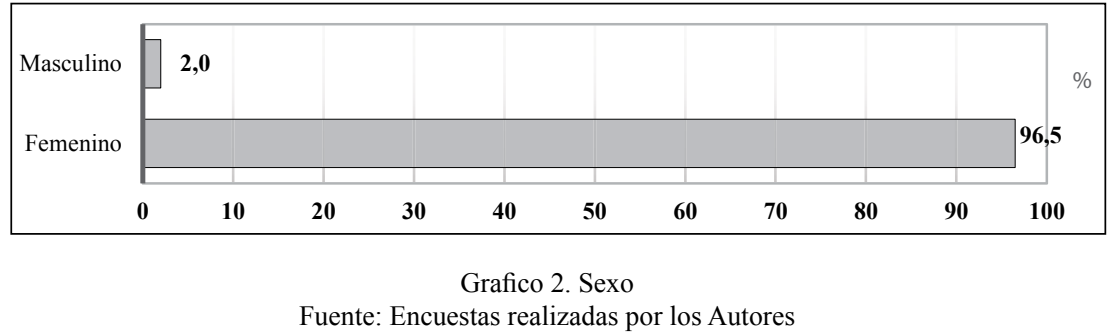

Se evidencia que el sexo predominante en los estudiantes de enfermería de la universidad de Barranquilla fue el femenino, considerando que esta profesión desde su historia en mayor porcentaje es estudiada por mujeres. 
Herminia Rosa Castellón-Montenegro, Cecilia Rocha-Rodríguez, Lina Montes-Rodríguez, Gloria Elena Lastre-Amell

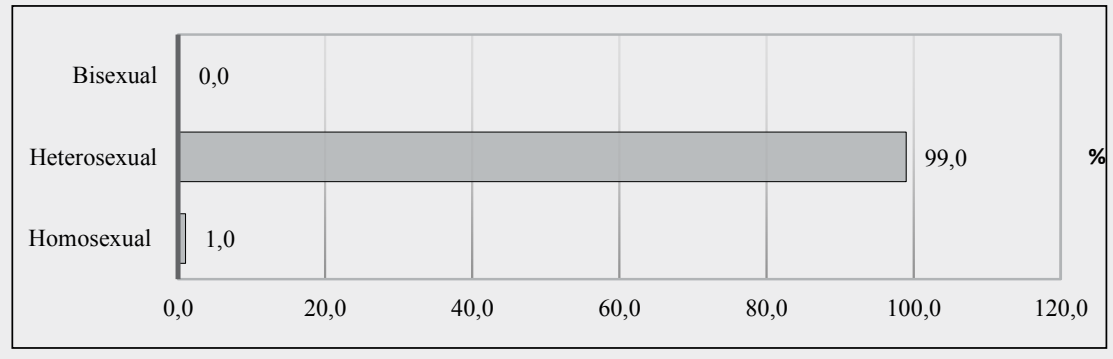

Grafico 3. Preferencia sexual

Fuente: Encuestas realizadas por los Autores

En contraste con la preferencia sexual de los estudiantes de enfermería se demostró que solo el $1 \%$ se declara homosexuales, anotando que el $99 \%$ de los estudiantes son heterosexual.

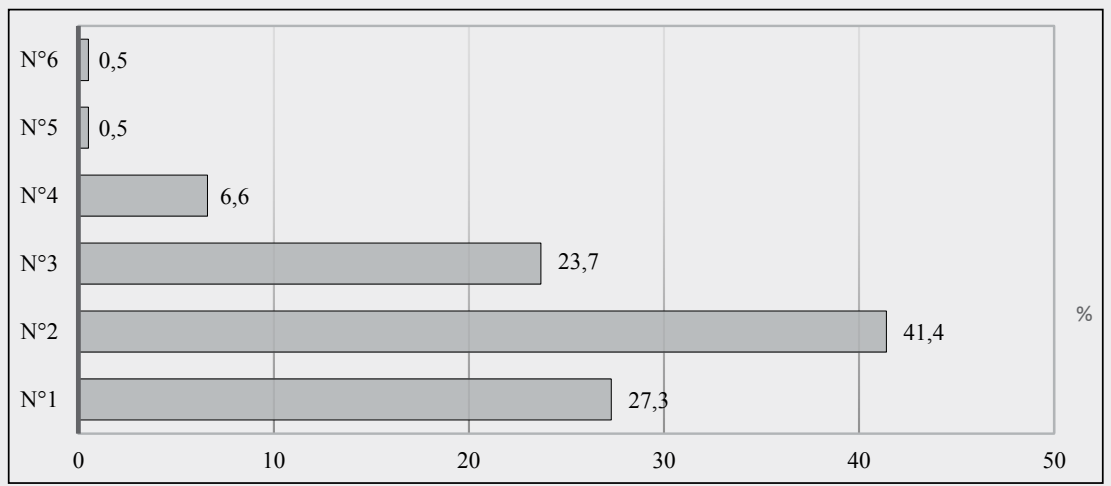

Grafico 4. Estrato socioeconomico

Fuente: Encuestas realizadas por los Autores

Del total de la población del estudio de conocimiento, actitud y prácticas del consumo de sustancias psicoactivas se encontró que el $41.4 \%$ pertenece a estrato socioeconómico 2 , seguido del 1 y el 3 .

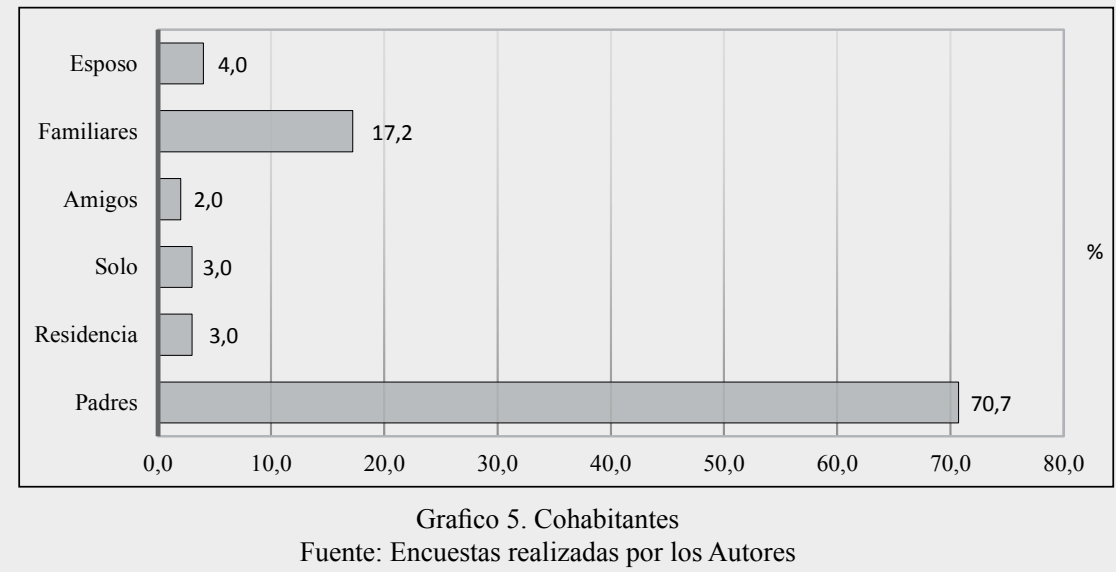

Se puede notar que el $70 \%$ de los estudiantes de enfermería encuestados viven con sus padres, lo que constituye en un factor protector denominado cohesión que favorece los procesos de formación integral y fundamentación de principios y valores indestronables. Se debe mencionar que un 3\% de ellos manifiesta vivir solo o en residencia puesto que durante su época de estudios permanecen pensionados en casas destinadas para estos fines. 
Respecto a los conocimientos de los estudiantes frente a la problemática de las sustancias psicoactivas encontramos:

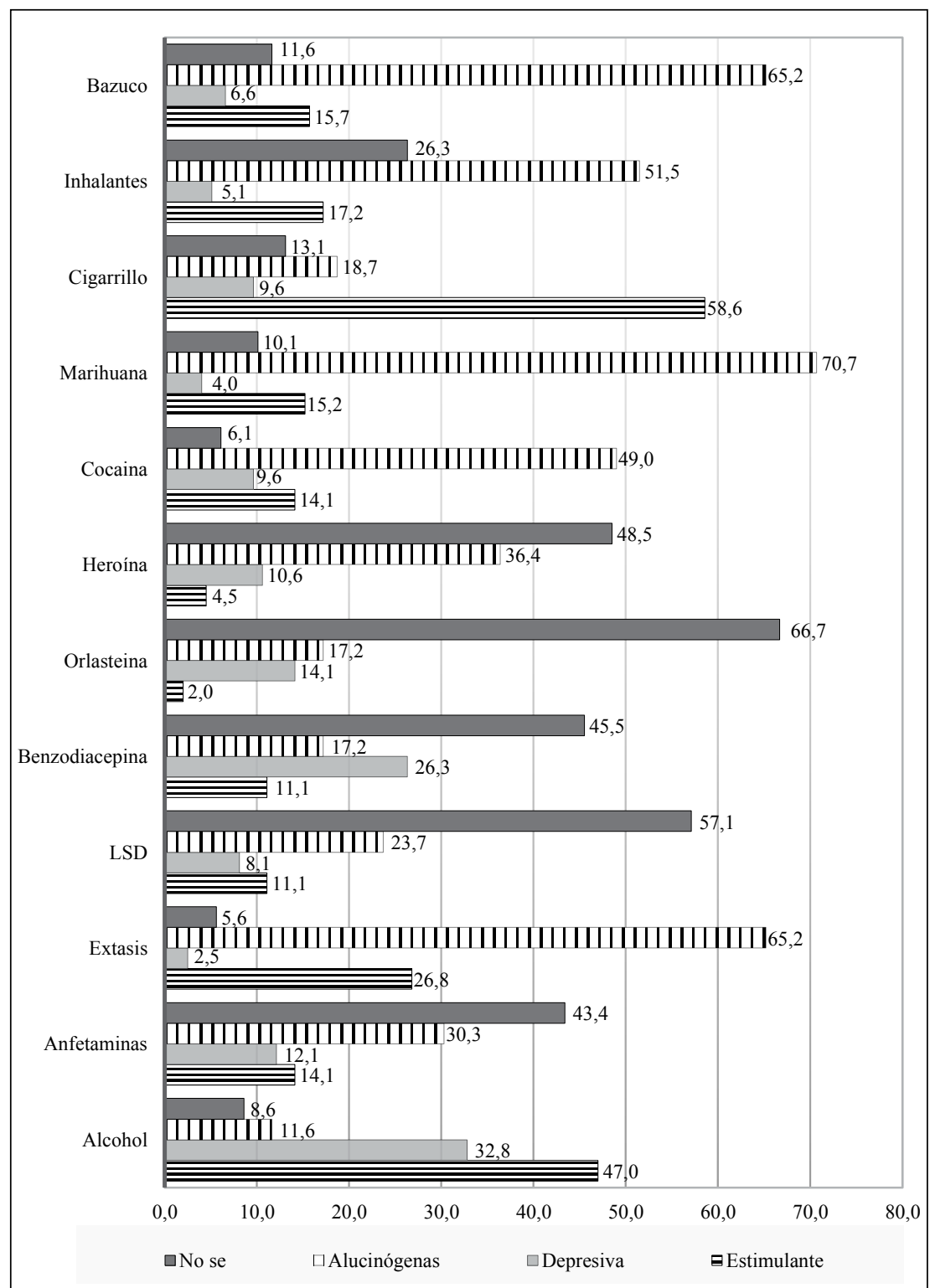

Grafico 6. Conocimiento acerca de sustancias psicoactivas Fuente: Encuestas realizadas por los Autores

En el estudio se observó que el $32.8 \%$ de los estudiantes de enfermería identificaron que el alcohol es una sustancia psicoactiva depresora, seguido del $23,7 \%$ de LSD es alucinógena, y el $15.7 \%$ respondieron que el bazuco y el $14.1 \%$ cocaína y anfetaminas lo identificaron como sustancia psicoactiva estimulante. 
Herminia Rosa Castellón-Montenegro, Cecilia Rocha-Rodríguez, Lina Montes-Rodríguez, Gloria Elena Lastre-Amell

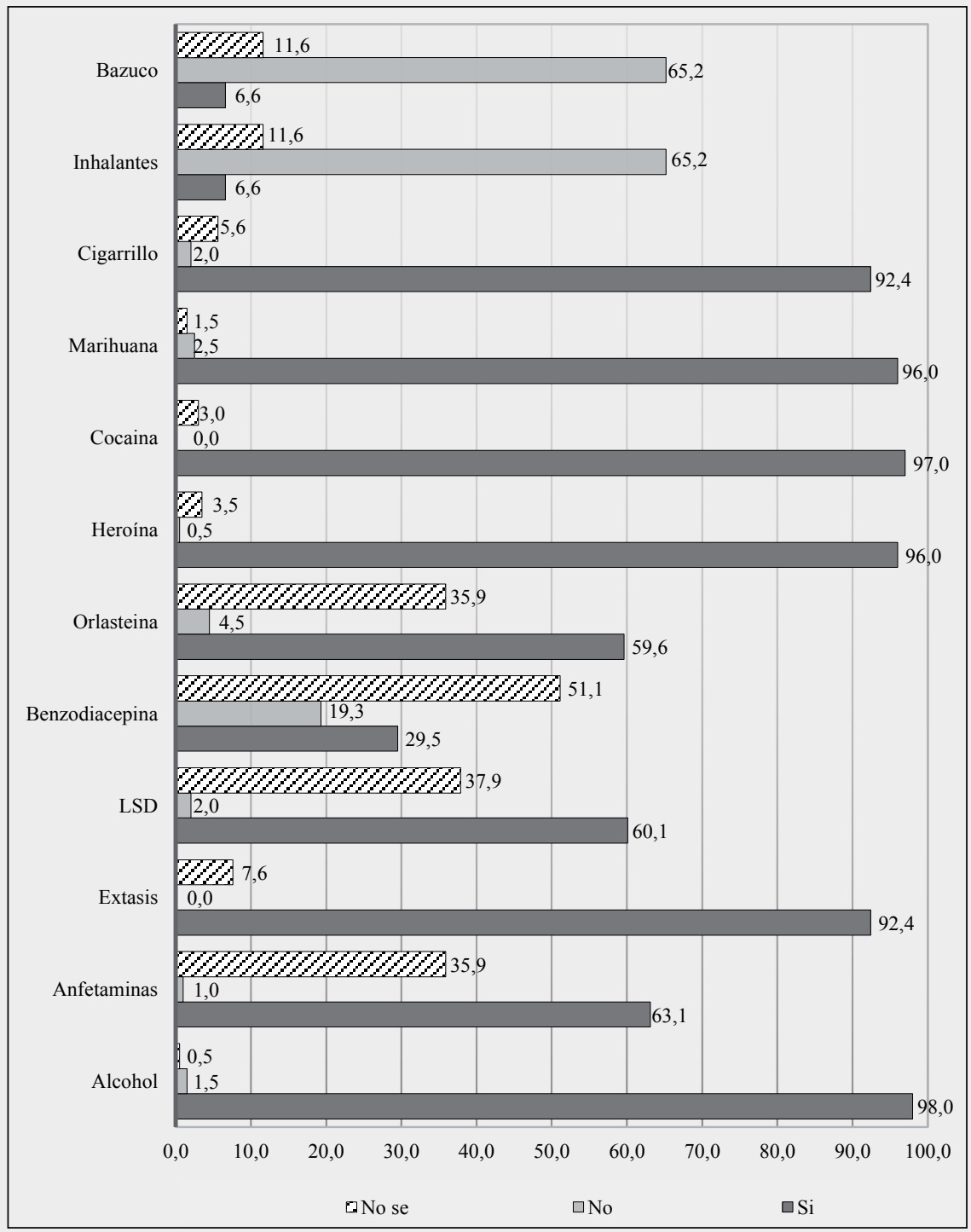

Grafico 7. SPA y sus efectos sobre la salud Fuente: Encuestas realizadas por los Autores

\section{Respuestas}

Cúcuta-Colombia

Vol. 20

No. 1

Enero - Junio 2015 ISSN 0122-820X

PP: $67-83$

Se muestra que el $98 \%$ de los estudiantes "SI" identificaron que el alcohol tiene efecto perjudicial para la salud, seguido del $97 \%$ de cocaína, $96 \%$ heroína, y el 92,4\% el éxtasis y el cigarrillo. Y el $65.2 \%$ "NO" considera perjudicial para la salud los inhalantes y el bazuco.

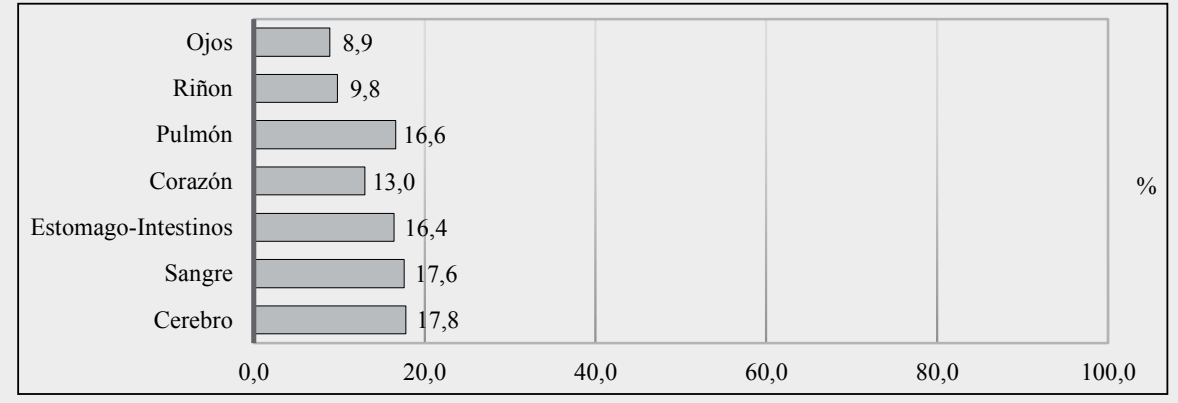

Grafico 8. Organos afectados por el consumo de SPA

Fuente: Encuestas realizadas por el Autor 
Conocimientos, actitudes y prácticas del consumo de sustancias psicoactivas en estudiantes de enfermería de una Universidad privada de Barranquilla 2010-2011

De acuerdo al Grafico 8 los estudiantes de enfermería de la universidad de Barranquilla se identificó que el Cerebro, la sangre y los pulmones son aquellos órganos del cuerpo que más sufre los efectos de las sustancias psicoactivas.

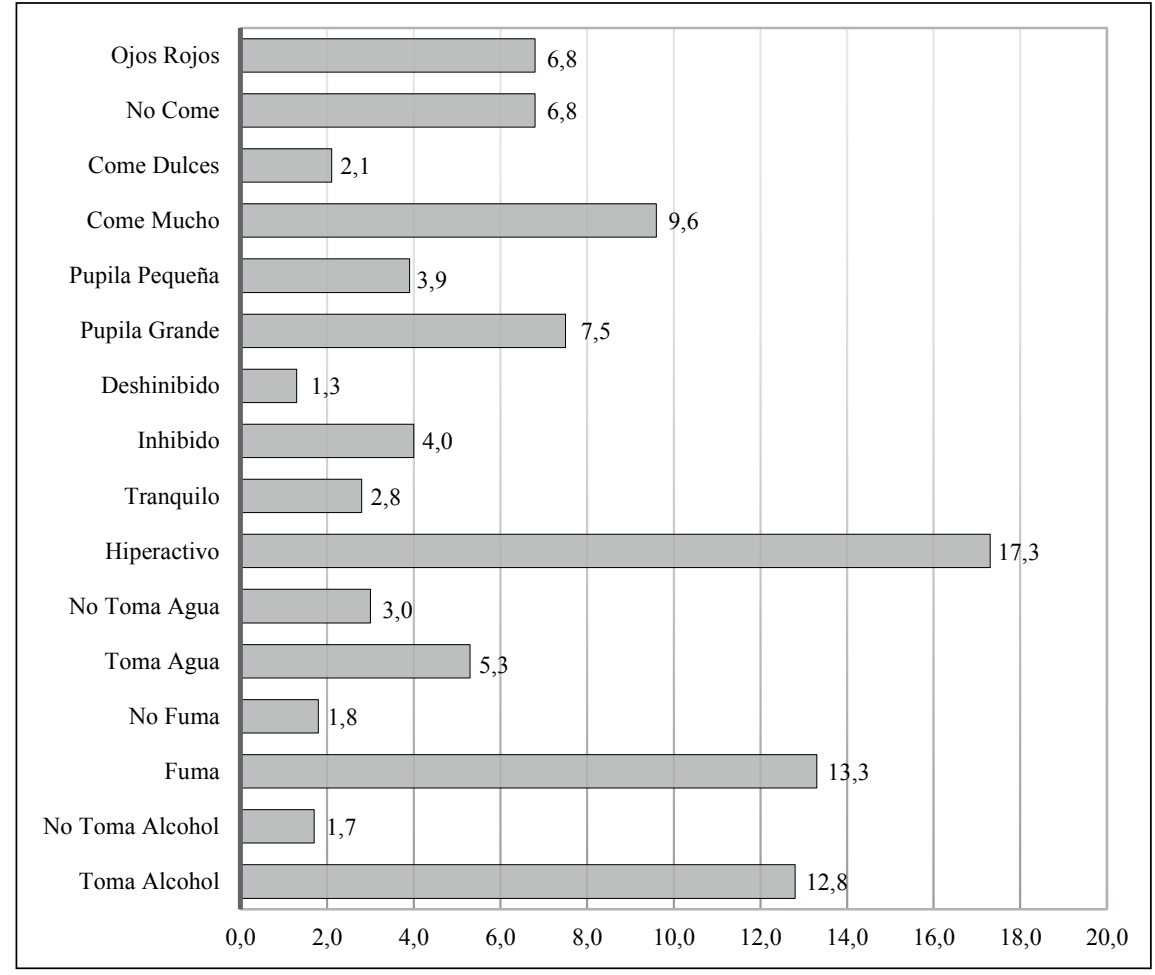

Grafico 9. Caracteristicas de consumidores de SPA

Fuente: Encuestas realizadas por el Autor

La mayoría de ellos coincide en que la hiperactividad y el aumento en la ingesta de alcohol son las características destacadas de los consumidores de SPA superando el 10\%.

Respecto a las Actitudes de los estudiantes frente a la problemática desatada por las SPA encontramos

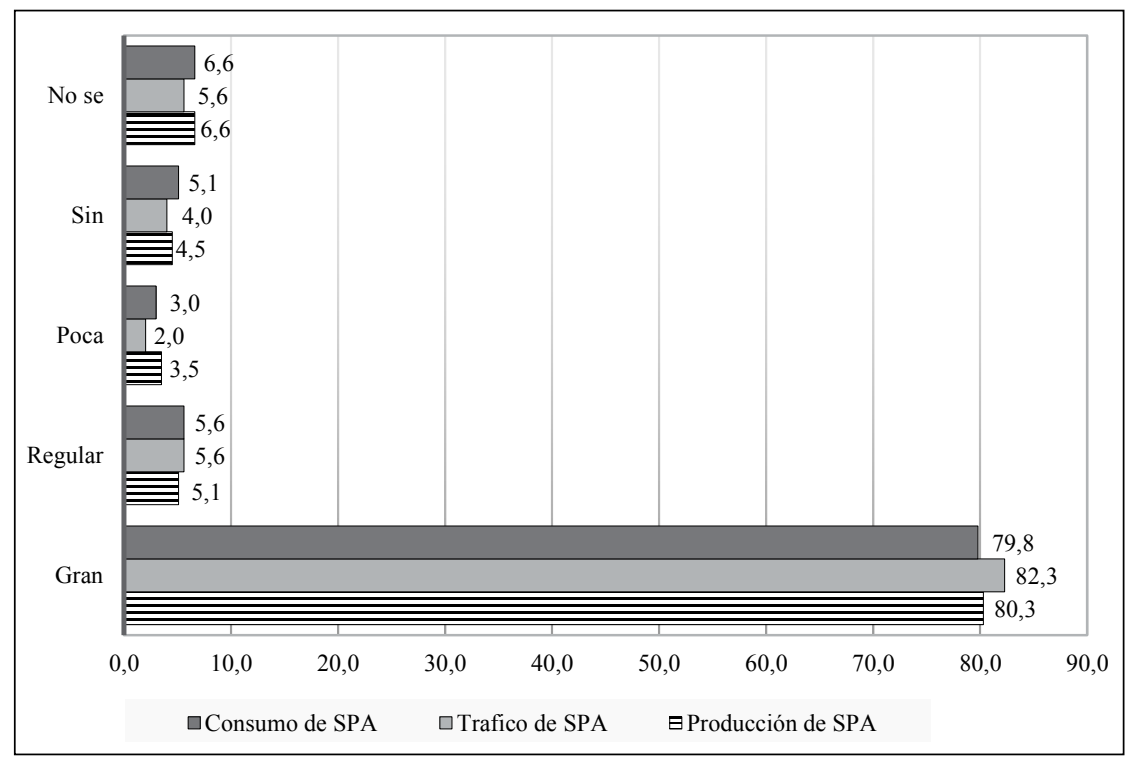

Grafico 10. Importancia sobre consumo, trafico y produccion de SPA Fuente: Encuestas realizadas por el Autor 
Se evidencio que el $80 \%$ de los estudiantes opinaron de gran importancia que se trate las temáticas de consumo, tráfico y producción de SPA, lo que nos arroja un resultado positivo dada la esencia de la profesión de enfermería que es el cuidado, y temáticas como estas, deben percibirse como importantes, porque somos profesionales de la salud y las drogas inciden y prevalecen con más frecuencia entre nuestra sociedad.

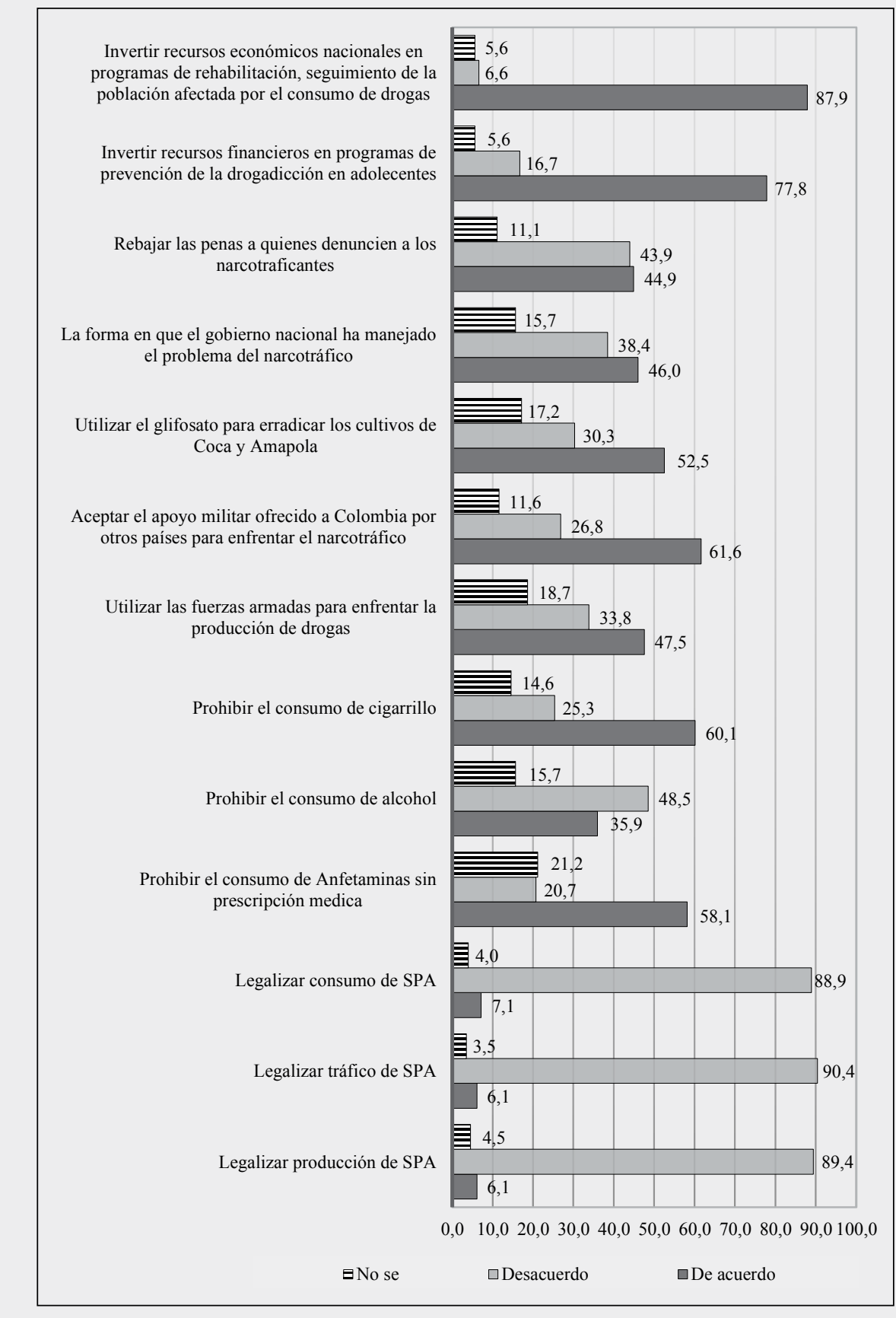

Enero - Junio 2015

Grafico 11. Actitudes

Fuente: Encuestas realizadas por el Autor

En general el $88 \%$ de los estudiantes rechazan todo tipo de intencion de legalizar la produccion, trafico o consumo de SPA, y apoyan todo esfuerzo del gobierno en erradicarlas y establecer programas que frenen su consumo. 
No. 1

Enero - Junio 2015 ISSN 0122-820X

PP: 67-83

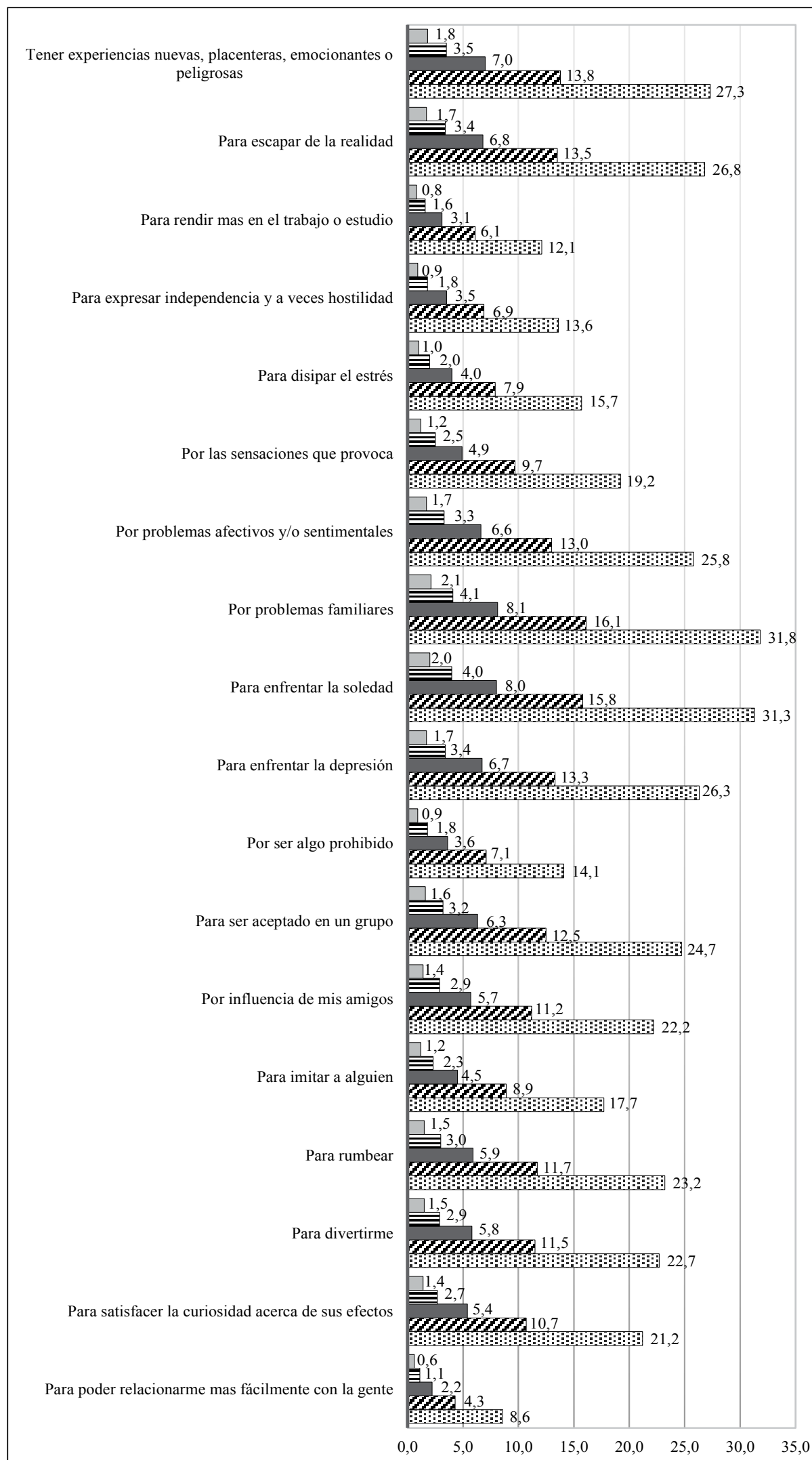

$\square$ Totalmente en desacuerdo $\boxminus$ Desacuerdo en parte $\square$ No tengo opinión $\square$ De acuerdo en parte $\boxminus$ Totalmente de acuerdo 
Para escapar de la realidad, ser aceptado en un grupo y por la influencia de los amigos superan el $56 \%$ de las opiniones de los estudiantes respecto a las razones por las cuales se inicia el consumo de drogas. Estuvieron muy equilibradas las demas respuestas.

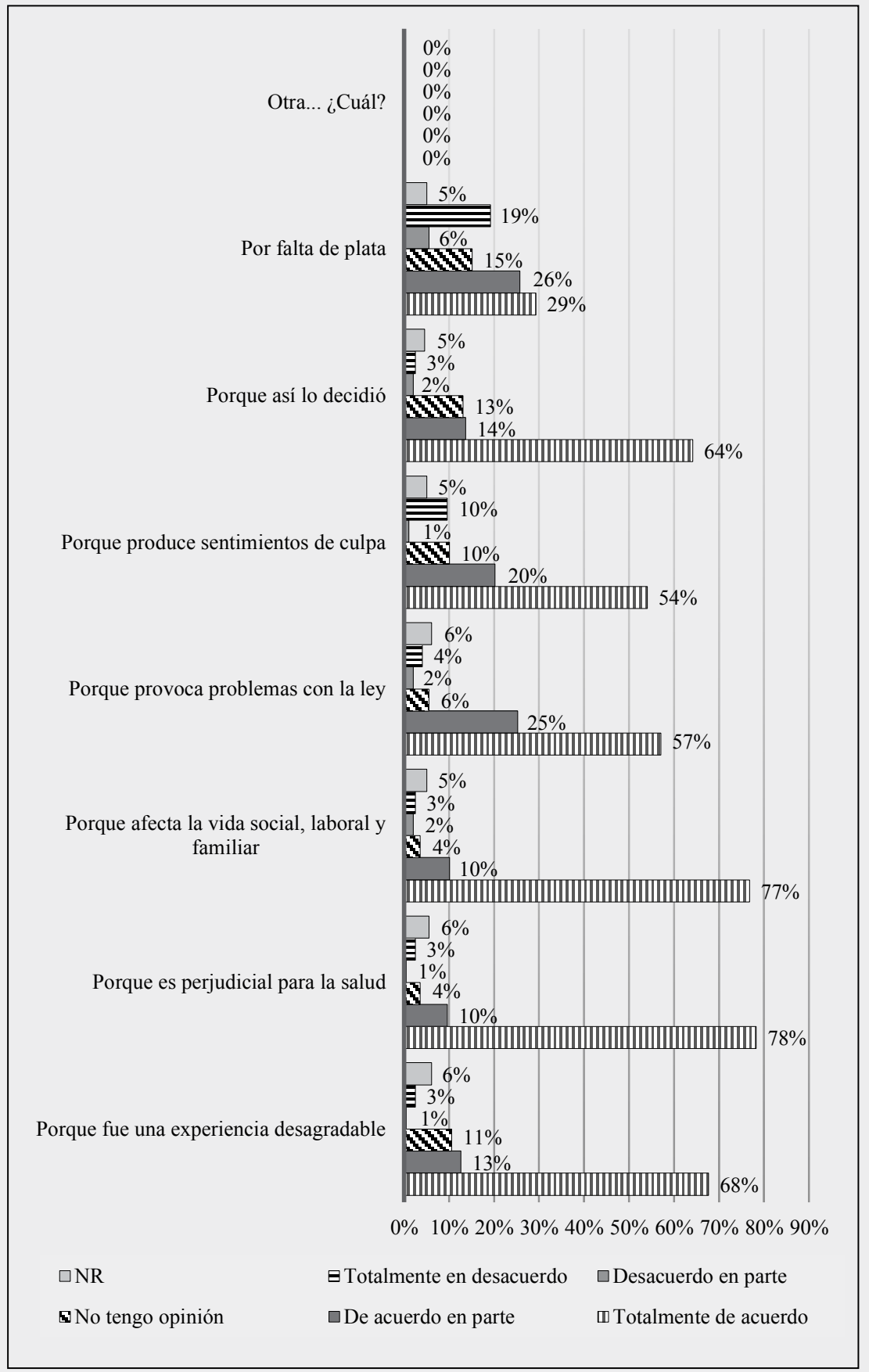

Grafico 13. Razones para dejar el consumo de SPA

Fuente: Encuestas realizadas por el Autor

Mas del 70\% de los estudiantes opina que aquellos consimidores que se apartan de las drogas lo hacen porque recapacitan frente al perjuicio que ocasiona sobre su salud. Superando el $76 \%$ de las respuestas ellos opinan que la rehabilitacion se produce motivados por la afectacion sobre la vida laboral, familiar y social. 
No. 1

Enero - Junio 2015 ISSN 0122-820X

PP: $67-83$

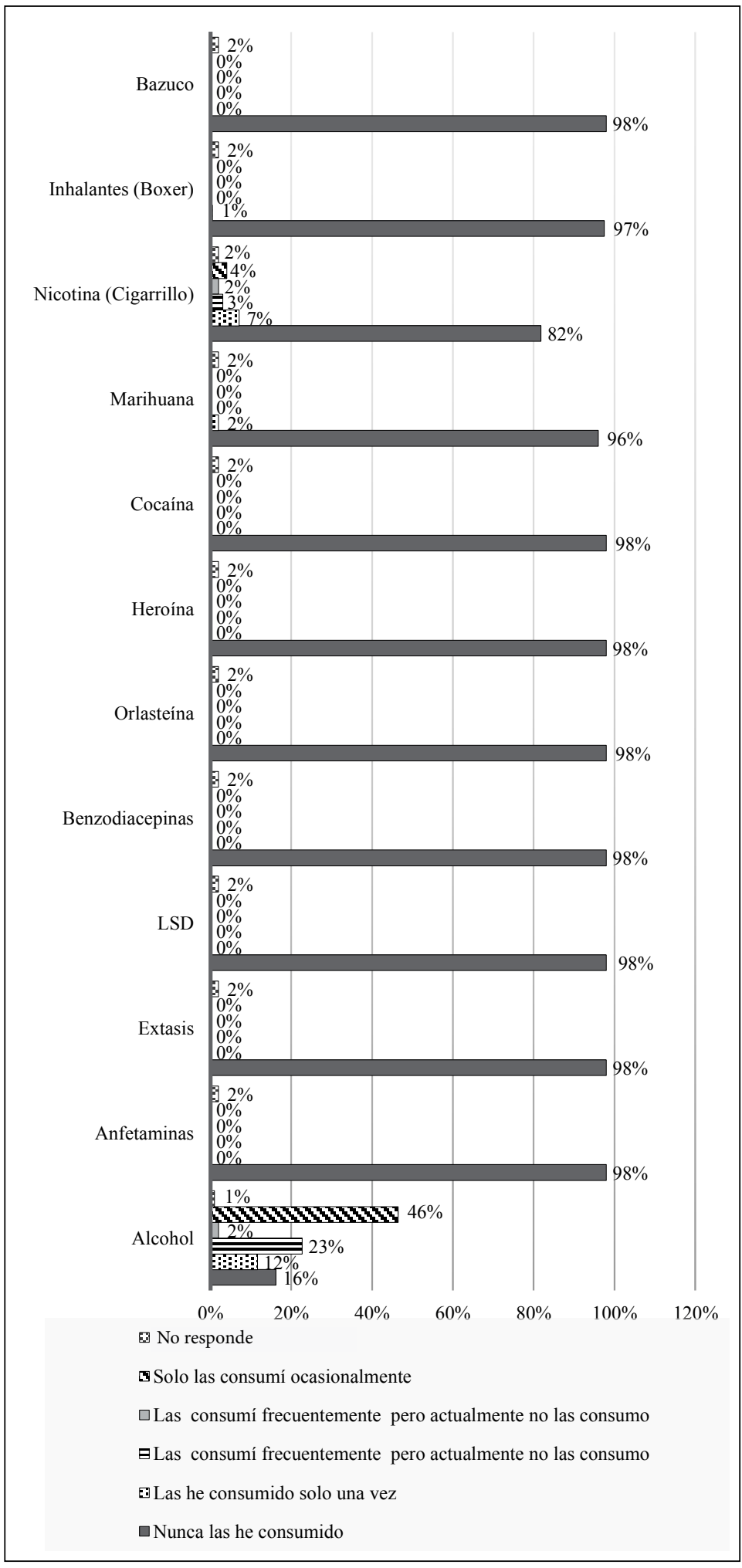

Grafico 14. Practicas de consumo de SPA

Fuente: encuestas realizadas por el autor

Aproximadamente un $97 \%$ de los estudiantes no han hecho uso de sustancias psicoactivas, a excepción del alcohol y nicotina; El 12\% de los estudiantes manifiesta haber consumido alcohol al menos una vez, y la nicotina el $9 \%$ de ellos. Otro resultado importante es que 5 estudiantes 
respondieron haber aceptado consumir alguna vez droga, 2 afirmaron que su inicio fue a los 14 años, 2 a los 20 y 1 a los 21; siendo la causa principal para su consumo por diversión, 4 estudiantes, y 1 por presión social y la droga usada fue la marihuana.

\subsection{Discusión}

En la investigación realizada sobre conocimiento, actitudes y prácticas del consumo de sustancias psicoactivas en los estudiantes de enfermería de una universidad de Barranquilla los resultados sociodemográficos obtenidos, se identificó que el $56.1 \%$ de los estudiantes encuestados se situó dentro del rango de edades comprendido entre los 18 y 20 años. Según lo expresado en [6], donde el consumo de sustancias adictivas es un problema socio sanitario de primer orden. Aunque la adolescencia es una etapa crítica para la adopción de pautas de abuso.

Además, se evidenció que el $96.5 \%$ son de sexo femenino y el $2 \%$ del sexo masculino. Teniendo en cuenta que la población universitaria es una comunidad joven y la mayoría son del sexo femenino, considerando esta profesión de mujeres así como lo data la historia y las "Herencias recibidas por tradición". Influencia española en la enfermería colombiana que estas herencias tienen que ver con la tradición de tipo religioso, la relación de la enfermería con la condición femenina o de género [7].

En contraste con la preferencia sexual de los estudiantes de enfermería se demostró que solo el $1 \%$ se declara homosexuales, anotando que el $99 \%$ de los estudiantes son heterosexual. Del total de la población del estudio de conocimiento, actitud y prácticas del consumo de sustancias psicoactivas se encontró que el $41.4 \%$ pertenece a estrato socioeconómico 2 , seguido del 1 y el 3 . Se puede notar que el $70 \%$ de los estudiantes de enfermería encuestados viven con sus padres, lo que se considera un factor protector denominado cohesión, que favorece los procesos de formación integral y fundamentación de principios y valores indestronables.

En cuanto a la variable de conocimiento sobre la clasificación de las sustancias si esta son estimulantes, depresoras y alucinógenas, se observó que el $32.8 \%$ de los estudiantes de enfermería identificaron que el alcohol es una sustancia psicoactiva depresora, seguido del $23,7 \%$ de LSD es alucinógena, el $15.7 \%$ respondieron que el bazuco, el $14.1 \%$ cocaína y anfetaminas lo identificaron como sustancia psicoactiva estimulante, lo que evidencia que la población de estudio si tienen conocimientos sobre las sustancias psicoactivas, lo que se contrasta con los resultados en [8], donde la población de estudio no pudo clasificar las sustancias adictivas que son las drogas psicotrópicas estimulantes y depresoras, según su uso socio legal, registrando que son drogas psicotrópicas el $82 \%$ de estudiantes universitarios. Por otra parte el conocimiento de la temática de las sustancias psicoactivas es importante mantener la enseñanza en las políticas y programas de enfermería a nivel nacional e internacional; así como lo referencia las acciones y líneas que marcan la Salud Internacional y el Fenómeno de las Drogas, como una fortaleza para mejorar la intervención en la área de salud [8].

Según la Organización Mundial de la Salud, estimó la contribución del consumo de alcohol, tabaco y sustancias ilícitas a la carga mundial de morbilidad (CMM). El proyecto reveló que las drogas licitas (tabaco y alcohol) son causas de mortalidad y discapacidad en los países desarrollados, y que es de esperar que el impacto del tabaco aumente en otras partes del mundo. No obstante, los resultados vuelven a destacar que la mayor parte de carga de morbilidad mundial se debe a las sustancias licitas, más que a las ilícitas [9]. Coincidiendo estos resultados con el estudio de los estudiantes de
Enero - Junio 2015 ISSN 0122-820X PP: $67-83$ 
enfermería de la universidad de Barranquilla donde ellos "SI" identificaron que el alcohol, la cocaína, heroína, éxtasis y el cigarrillo tiene efecto perjudicial para la salud, Y el $65.2 \%$ "NO" considera perjudicial para la salud los inhalantes y el bazuco, teniendo en cuenta que las sustancias psicoactivas licitas e ilícitas alteran los estados de conciencia, de ánimo y de pensamiento, considerando así un problema de salud pública.

Con respecto a la variable de órganos afectados se identificó que el cerebro, la sangre y los pulmones son los sistemas del cuerpo que más sufre los efectos de las sustancias psicoactivas, coincidiendo este resultado con el estudio de Montalvo Prieto y Castillo Ávila sobre conocimientos, actitudes y prácticas frente a las sustancias lícitas e ilícitas de los estudiantes de enfermería de Cartagena, donde mostraron que el cerebro (94\%) es el órgano más afectado por el consumo de estas sustancias [10]. Afianzando este concepto con lo expresado en [11], donde en las civilizaciones siempre han existido registros de diversos tipos de uso de SPA que alteran el funcionamiento del sistema nervioso central.

En este mismo orden de ideas, en [11] se identificaron que las organizaciones internacionales despliegan esfuerzos para el control, producción, comercialización y consumo de las SPA; también han involucrado a los gobiernos a que exista control, que incluyan programas de financiamiento, cooperación técnica, entidades educativas y de salud y recursos humanos para el control del fenómeno. Ante este aspecto se demostró en el estudio con relación a las actitudes que tuvieron los estudiantes frente al tráfico y producción de las SPA se evidenció que el $80 \%$ opinaron que el tema es de gran importancia y mostraron preocupación que se trate estas temáticas, lo que nos arroja un resultado positivo dada la esencia de la profesión de enfermería que es el cuidado y temáticas como estas deben percibirse importantes, porque somos profesionales de la salud y las drogas inciden y prevalecen con más frecuencia entre nuestra sociedad.

En general el $88 \%$ de los estudiantes rechazan todo tipo de intencion de legalizar la produccion, trafico o consumo de SPA, $\mathrm{y}$ apoyan todo esfuerzo del gobierno en erradicarlas y establecer programas que frenen su consumo. A pesar que Colombia en los años 2003 presentaba estas variables y los organismos internacionales trabajan por esta problemática como La Organización de los Estados Americanos (OEA) ha asumido un rol importante en la lucha de las drogas en el continente. Y no solo para cumplir con el papel creó tres instancias especializadas: la Comisión Interamericana para el Control del Abuso de Drogas (CICAD), el Observatorio Interamericano sobre Drogas (OID) y la Red Interamericana de Telecomunicaciones para el Control de Drogas (RETCOD) [12]. Cuyo objetivo de la CICAD fue eliminar el tráfico ilícito y el uso indebido de drogas, y en el "Informe Mundial sobre las Drogas 2012" se evidencia motivos de preocupacion sobre la producción, el tráfico y el consumo de drogas ilícitas [13].

De acuerdo a las practicas del consumo de SPA en la investigación se encontró que aproximadamente un $97 \%$ de los estudiantes no han hecho uso de sustancias psicoactivas, a excepción del alcohol y nicotina; siendo el alcohol consumido al menos una vez el 12\%, y la nicotina el $9 \%$ de ellos. Cabe de anotar que el alcohol es consumido actualmente por el $46 \%$ y el cigarrillo el $7 \%$. Otro resultado importante es que 5 estudiantes respondieron haber aceptado consumir alguna vez droga, 2 afirmaron que su inicio fue a los 14 años, 2 a $\operatorname{los} 20$ y 1 a $\operatorname{los} 21$; siendo la causa principal para su consumo por diversión, 4 estudiantes, y 1 por presión social y la droga usada fue la marihuana; corroborado estos resultados con estudios anteriores, relacionados a las drogas legales, la Organización Mundial de 
la Salud afirma que el consumo de alcohol es calificado el tercer factor de riesgo en los países industrializados y el principal en los países en desarrollo, y en el mismo estudio sobre Consumo de drogas en jóvenes universitarios y su relación de riesgo y protección con los factores psicosociales [14], según las Características del consumo de drogas ilegales y legales muestra que el alcohol $(74,5 \%)$, tabaco $(33,2 \%)$, siendo el alcohol en la mayoría de los jóvenes iniciando su consumo entre los 12 y 17 años $(84,3 \%)$, y es la sustancia más consumida entre los universitarios: el $97,4 \%$ ha tomado al menos una vez en su vida. Razón por la cual sabemos que las drogas legales son las puertas de entrada para el inicio de consumir SPA en los adolescentes [14]. No obstante las drogas como el alcohol y el tabaco en el Informe Mundial sobre las drogas revelan que la prevalencia anual del consumo del alcohol es del $42 \%$, y del tabaco el $25 \%$ de la población mayor de 25 años, considerando que estas SPA son legales en la mayoría de los países [13].

Al revisar estudios referentes a razones de consumo de SPA en adolescentes se identifico en la investigacion de Factores de riesgo relacionados al uso de drogas ilegales: perspectiva crítica de familiares y personas cercanas en un centro de salud público en San Pedro Sula, Honduras, en donde encontraron que los factores de presión social correspondidos al uso de drogas, son situación familiar o social, falta de conocimiento y de comprensión sobre las drogas, la presión de los amigos o amigas y el tener amistades que usan drogas; aspecto que coincide con el estudio de los estudiantes de enfermeria de la universidad de Barranquilla fue "escapar de la realidad", "ser aceptado en un grupo" y "por la influencia de los amigos" razones por las cuales se han demostrado que los jovenes inicia el consumo de drogas [15].

\section{Conclusiones}

Este estudio revela el importante papel que desempeñan los programas y escuelas de enfermería frente la problemática de consumo de SPA, en la medida que los semestres son más complejos se observa un notable cambio de actitud y un nivel de conocimientos superior. La tarea de la educación en SPA es vital para concertar acciones interinstitucionales, interdisciplinares e intersectoriales para hacer promoción, prevención, tratamiento, rehabilitación y reinserción social de sujetos vulnerables y consumidores de sustancias psicoactivas en nuestra región [16].

Algunas de las principales conclusiones resaltan el papel de los proyectos de intervención que desarrolla el personal de enfermería:

- Educar a los adolescentes en la Prevención de Sustancias Psicoactivas, para disminuir el alto nivel de consumo, el riesgo de enfermedades y mortalidad de los estudiantes.

- Fortalecer el componente de comunicación.

- Propiciar la participación de la comunidad.

- Conformar equipos interdisciplinarios que coordinen las actividades de la atención integral [16].

Es importante que los padres de familia, maestros y personas mayores, amigos $\mathrm{o}$ familiares de niños y jóvenes que noten cualquier cambio en su conducta y en sus hábitos o que conozcan abiertamente que exista esta adicción, traten de convencer y/o soliciten ayuda, para que los potenciales consumidores puedan recibir atención oportuna y adecuada a su problema [17].

Crear un sistema de capacitación permanente, continua y sostenible, a través de convenios con fundaciones, universidades o instituciones del Estado, orientado al personal que ejerza funciones de control, prevención, represión y tratamiento, relacionado con las nuevas sustancias o con las drogas sintéticas [17]. 5.
Enero - Junio 2015 ISSN 0122-820X PP: $67-83$ 


\section{Agradecimientos}

Un especial saludo de agradecimiento a todos los estudiantes de enfermería y docentes acompañantes que aportaron tiempo $\mathrm{y}$ espacio para contribuir en la adquisición de conocimientos importantes que nos impulsan a desarrollar labores de intervención que contribuyan a nivel local, regional y nacional en la prevención del consumo de SPA.

De igual manera agradecemos a La Comisión Interamericana para el Control del Abuso de Drogas (CICAD) y a la Universidad privada de Barranquilla por los recursos suministrados para la realización de este proyecto.

\section{Referencias}

[1] A.Carballeda.La farmacodependencia en América Latina: su abordaje desde la atención primaria de salud. Washington: Organización Panamericana de la Salud; 1991.

[2] Ministerio de la protección social (MPS), Dirección Nacional de Estupefacientes (DNE), Oficina de las Naciones Unidas contra la Droga y el Delito (UNODC), Comisión Interamericana para el Control del Abuso de Drogas (CICAD) Organización de los Estados Americanos (OEA), Embajada de los Estados Unidos en Colombia. Estudio nacional de consumo de sustancias psicoactivas en Colombia - 2008. Bogotá D.C., [Online]. Disponible en: https://www.unodc.org/documents/ colombia/2013/septiembre/ Estudio Nacional_Consumo_2008.pdf.

[3] J. F. Cepeda, G. Pezzano, M. Racedo. Prevengamos la farmacodependencia: Programas educativos. Bogotá: Uninorte; 1989.
[4] Ministerio de Salud. Normas científicas, técnicas y administrativas para la investigación en salud. Resolución 008430 del 4 de octubre de 1993. Santafé de Bogotá D.C. [Online]. Disponible en: http://www.minsalud.gov.co/ Normatividad /RESOLUCION \%20 8430\%20DE\%201993.pdf .

[5] Ministerio del Congreso. Disposiciones en materia de responsabilidad deontológica para el ejercicio de la profesión de Enfermería en Colombia. Ley 911 del 4 de octubre de 2004. Diario Oficial, no. 45.693.

[6] E. Martín, F. J. Barón, L. O. Rubio, et al, "Consumo de alcohol, tabaco, cannabisyotras sustanciaspsicoactivas en estudiantes de la Universidad de Málaga", Rev Trastornos Adictivos, vol. 13, no. 4, pp.160-166, 2011.

[7] A. L. Velandia, "Influencia española en la enfermería colombiana", Rev. Latino-Am. Enfermagem, vol. 1, no. 2, Jul 1993. [Online]. Disponible en: http://www.scielo.br/scielo. php?script $=$ sci_arttext\&pid=S0104$11691993000200011 \& \operatorname{lng}=$ en\&nrm =iso. DOI: http://dx.doi.org/10.1590/ S0104-11691993000200011.

[8] M. P. Navia, J. Farah, N. Yaksic, et al, "Conocimiento sobre el fenómeno de las drogas en entre estudiantes y docentes de la Facultad de Medicina Universidad Mayor de San Andrés, La Paz, Bolivia”, Rev. Latino-Am. Enfermagem, vol. 19, no. esp, pp. 722-729, Jun 2011. [Online]. Disponible en: http://www. scielo.br/scielo.php?script=sci artte xt\&pid=S0104-1692011000700009 \&lng=es\&nrm=iso. DOI: http:// dx.doi.org/10.1590/S010411692011000700009 . 
[9] Organización Mundial de la Salud. Neurociencia del consumo y dependencia de sustancias psicoactivas Ginebra. 2004. [Online]. Disponible en: http://www.who.int/ substance_abuse/publications/en/ Neuroscience_S.pdf.

[10] A. Montalvo, I. Y. Castillo, "Knowledge, attitudes, and practices regarding legal and illegal substances by nursing students from Cartagena". Invest Educ Enferm, vol. 31, no. 1, pp. 63-69, 2013.

[11] N. Rebolledo, M. C. Costa, "Significados y contradicciones del fenómeno de las drogas: drogas lícitas e ilícitas en Chile", Rev. Latino-Am. Enfermagem, vol. 13, no. esp, pp. 903911, Oct 2005. [Online]. Disponible en: http://www.scielo.br/scielo. php? script $=$ sci_arttext\&pid=S010411692005000700019\&lng=en. DOI: http://dx.doi.org/10.1590/S010411692005000700019.

[12] A. Vergara, Y. Lahuerta, S. P. Correa. Posibles implicaciones de la legalización del consumo, producción y comercialización de las drogas en Colombia. Documento 234. Sep 2003. [Online]. Disponible en: https:/colaboracion.dnp.gov.co/CDT/ Estudios\%20Econmicos/234.pdf.

[13] Naciones Unidas. Oficina contra la droga y el delito. Informe mundial sobre las drogas 2012. [Online]. Disponible en: http://www.unodc. org/documents/data-and-analysis/ WDR2012/Executive_summary_ spanish.pdf.

[14] D. Cáceres, I. Salazar, M. Varela, J. Tovar, "Consumo de drogas en jóvenes universitarios y su relación de riesgo y protección con los factores psicosociales", Univ. Psychol., vol. 5, no. 3, pp. 521534, 2006. [Online]. Disponible en: http://pepsic.bvsalud.org/scielo. php?script $=$ sci_arttext\&pid=S1657$92672006000300008 \& \operatorname{lng}=$ pt\&tlng $=$ en.

[15] G. M. Rodríguez, B. Brands, E. Adlaf, et al, "Factores de riesgo relacionados al uso de drogas ilegales: perspectiva críticadefamiliaresypersonas cercanas en un centro de salud público en San Pedro Sula", Honduras. Rev. LatinoAm. Enfermagem, vol. 17, no. spe, pp. 796-802, 2009. [Online]. Disponible en:http://www.scielo.br/scielo. php?script $=$ sci_arttext\&pid=S0104$11692009000700007 \& \operatorname{lng}=$ en. DOI: http://dx.doi.org/10.1590/S010411692009000700007.

[16] Organización Panamericana de la Salud. Protocolo de prevención en farmacodependencia para el niño escolar y adolescente. Programa de prevención en farmacodependencia. [Online]. Disponible en: http:// www.col.ops-oms.org/Municipios/ Cali/10FarmacoDependencia.htm.

[17] A. M. Bedoya y C. G. Oviedo. Ensayo propositivo: "Alerta por consumo de nuevas sustancias psicoactivas". Policía Nacional, Bogotá, Colombia, 2008. [Online]. Disponible en: http:// www.policia.gov.co/portal/page/ portal/UNIDADES_POLICIALES/ Escuelas_formacion/sespo/ LA_ESCUELA/AREAS/AREA INVESTIGACION/PROCESOS INVEST/INVESTIGACION_ F O R M AT IVA / A L E R T A \% 20 POR $\% 20 C O N S U M O \% 20 D E \% 20$ NUEVAS $\% 20$ SUS TANCIAS $\% 20$ PSICOACTIVAS.pdf. 